\title{
EEG based Brain Alertness Monitoring by Statistical and Artificial Neural Network Approach
}

\author{
Md. Asadur Rahman ${ }^{1}$, Md. \\ Mamun or Rashid ${ }^{2}$ \\ Department of Biomedical \\ Engineering, Khulna University of \\ Engineering \& Technology (KUET) \\ Khulna-9203, Bangladesh
}

\author{
Farzana Khanam $^{3}$ \\ Department of Biomedical \\ Engineering, Jessore University of \\ Science and Technology (JUST), \\ Jessore-7408, Bangladesh
}

\author{
Mohammad Khurshed Alam ${ }^{4}$, \\ Mohiuddin $\mathrm{Ahmad}^{5}$ \\ Department of Electrical and \\ Electronic Engineering, Khulna \\ University of Engineering \& \\ Technology (KUET), Khulna-9203, \\ Bangladesh
}

\begin{abstract}
Since several work requires continuous alertness like efficient driving, learning, etc. efficient measurement of the alertness states through neural activity is a crucial challenge for the researchers. This work reports a practical method to investigate the alertness state from electroencephalography (EEG) of the human brain. Here, we have proposed a novel idea to monitor the brain alertness from EEG signal that can discriminate the alertness state comparing resting state with a simple statistical threshold. We have investigated two different types of mental tasks: alphabet counting \& virtual driving to monitor their alertness level. The EEG signals are acquired from several participants regarding alphabet counting and virtual motor driving tasks. A 9-channel wireless EEG system has been used to acquire their EEG signals from frontal, central, and parietal lobe of the brain. With suitable preprocessing, signal dimensions are reduced by principal component analysis and the features of the signals are extracted by the discrete wavelet transformation method. Using the features, alertness states are classified using the artificial neural network. Additionally, the relative power of responsible frequency band to alertness is analyzed with statistical inference. We have found that the beta relative power increases at a significant level due to alertness which is good enough to differentiate the alertness state from the control state. It is also found that the increment of beta relative power for virtual driving is much greater than the alphabet counting mental alertness. We hope that this work will be very helpful to monitor constant alertness for efficient driving and learning.
\end{abstract}

Keywords-Alertness monitoring; Electroencephalography (EEG); Principal Component Analysis (PCA); Analysis of Variance (ANOVA); Discrete Wavelet Transformation (DWT); Band Relative Power; Artificial Neural Network (ANN)

\section{INTRODUCTION}

Brain functionality is directly related to its electrical activity because the neurons communicate with each other based on their functioning. This electrical activity can be measured from the scalp of the human brain by an electroencephalograph (EEG). The finer temporal resolution of EEG signal benefits scientific arena to exploit it in research and engineering. Recent developments [1-7] prove that different human activities can be recognized or classified by the EEG signal. Besides vast applications and implementations of EEG signal in the various fields (biomedical instrumentation, neuroscience, brain-computer interface, etc.), EEG signal can be utilized to detect alertness state with respect to resting state. According to neurophysiological methodology, drowsiness is considered as the transition from awaking state into sleeping state or deep relaxation state, marked by reduces alertness and slow movements. Sleeping state begins with the activation of neurons and brain inhibition. The transformation of awaking or alertness state to unconscious or drowsiness state is described by certain rhythmic changes [8-10]: (i) decreased the beta rhythmic (13-30 Hz) activity, (ii) increase in alpha rhythm activity $(8-13 \mathrm{~Hz})$ but best observable while resting by eyes closed; and (iii) increased theta rhythm activity $(4-8 \mathrm{~Hz})$ if consequently alpha rhythm decreased.

The classrooms can be modernized introducing wireless EEG system to monitor the attention level of the students regarding their mental capability of attentiveness. Furthermore, additional importance should pay to the drivers or pilots because still now on average 3,287 people die each day by road crush worldwide which can be largely reduced with the attention level monitoring system [11]. An alarm unit can be integrated to aware the drivers or pilots to remind their inattentions. This implementation of the EEG signal cannot be overlooked for the concern of road accidents reduction; consequently, innumerable lives can be saved. Plus, neurological disorder named by attention deficit hyperactivity disorder (ADHD) is often characterized by certain symptoms like inattention, impulsivity, and hyperactivity those create problems for patients to remember information, to concentrate, to arrange tasks, etc. Worldwide $5.9-7.1 \%$ of school-going children suffer from ADHD [12] and 30-50\% of them spotted in childhood endure to exhibit symptoms into later life [13-15]. Therefore monitoring constant consciousness of ADHD patient as well as the brain alertness monitoring of the driver are two major concerns and the sound monitoring of these two aspects can provide a positive impact on our social development.

Different types of research works regarding the aforementioned problems have been accomplished. In [16-23], researchers proposed a classification method of different states (attentive, inattentive alertness, sleepiness, drowsiness). Authors in [16] propose a method to make the learning process of students more effective by distinguishing attentive and inattentive state. Authors employed a portable brainwave 
sensor to collect the EEG data from the participants. Support vector machine (SVM) classifier is used after extracting various features to arrange a feature set to identify student's attentive state and provides $76.82 \%$ as highest classification accuracy. Authors in [17] introduced a method of separating alertness and sleepiness state for riskless driving using EEG signal as a reliable source. The features of EEG signals are classified by an artificial neural network (ANN) which results in $83.3 \%$ classification accuracy. In [18] authors suggested another work to detect the drowsiness of the driver where using wavelet, spectral, and time analysis, on average $85 \%$ classification accuracies are found by ANN. An automatic alertness detection system from three states (alert, drowsy and sleep) is proposed in [19]. Here, power spectral density (PSD) through discrete wavelet transform (DWT) is used as the feature and ANN classified the features with above $90 \%$ accuracy. Researchers in [20] proposed a sleep stage classification method for diagnosis purpose in psychiatry and neurology. The Physionet database exploited for data collection and wavelet packet tree (WPT) used to extracts features. Three classifiers are used to evaluate the accuracy rate and found overall accuracy $70 \%$. Another work classifies drowsiness with respect to alertness extracting energy coefficients from WT to train ANN and found $90.27 \%$ accuracy [21]. Authors in [22] classify participant's 3 states (alert, drowsy and sleep) DWT and ANN classifier which results in satisfactory accuracy rate. A drowsiness detection method of the driver due to mental fatigued using heart rate differences is proposed in [23]. Here, WT and Fast Fourier Transform (FFT) based features are selected and classified using SVM and finally compares accuracy which shows that WT based features provide better accuracy rate (95\%) than FFT based features. Authors in [24] developed a driver distraction level measurement method using different wavelets of WPT depending 4 distraction stimuli. Analyzing results from 3 classifiers, subtractive fuzzy inference system classifier and sym 8 wavelet provides best accuracy of $79.21 \%$ based on PSD feature.

Among the research works [16-24], it is observed that the classification accuracy rate is not convincing for all. Therefore there arises a scope to develop a method that can increase the classification accuracy of different mental alertness states. In addition to that, these works did not mention any statistical threshold level for the mental alertness states by which method highly alertness or non-alertness can be monitored practically while performing risky works. On the other hand, authors in [9, 25-26] proposed different feature selection methods to enhance the classification accuracy or overall detection performance. Authors in [9] describe a drowsiness detection system where the feature is selected based on the most responsible $\mathrm{m}$ terms approximation of the DWT expansion. Authors claimed that this method alleviates the use of complex techniques. In [25], researchers introduced a feature extraction method for mental multitask classification. Empirical wavelet transform (EWT) with fuzzy clustering method was employed for feature extraction. After feature selection, vectors are feed to support vector classifier (SVC). In another research, the article proposed a method to increase the performance of mental task using WT and EMD feature extraction method [26]. There are some other works those were performed special investigations such as a BCI application of patient monitoring by EEG signal of mental alertness [27], mental fatigue or workload comparing with alertness [28] and a drowsy driving monitoring technique [29]. These methods are very complicated and several features with classifier are used to determine alertness/active state.

From the literature, it can be concluded that most of the works classify alertness state or drowsiness state by extracting different features for various purpose to enhance the accuracy rate but it is noticeable that accuracy rate is not improved that much. So it is very important to delineate a method which would provide better accuracy for alertness classification. Additionally, still, no such indication is implied by any researchers to monitor the alertness continuously so that the method can monitor the subject performing risky tasks for avoiding error or accident such as driving. In this research work, we have proposed two methods for alertness assessments. One is for classifying mental alertness states of two significant mental tasks: alphabet counting and virtual driving with resting state (eyes open and eyes close) offering very high accuracy. Another method is to monitor the alertness of the person while performing virtual driving with respect to resting state which is based on a statistical threshold. Both methods are promising for alertness classification and monitoring. The main contribution of this research work is applying the suitable methods with proper signal processing steps to classify the different mental alertness states with a high degree of accuracy. In addition, another major contributing indication for continuous mental alertness monitoring is proposed based on the statistical threshold of band relative power.

The rest of the paper is prearranged as following: Section II describes the materials used in this research work and the mathematical methods applied in this research work. Section III presents the experimental results with their elaborate discussion. Finally, we have concluded our total research work in Section IV with future perspectives.

\section{MAterials And MATHEMATICAL Methods}

\section{A. Experimental Protocols}

Experimental protocols regarding this research work were arranged in such a way that we could be able to mimic the environment for monitoring the concentration of students or where requires constant concentration. In addition, another virtual atmosphere was created where the alertness of a driver or pilot could be monitored which will be adopted to reduce the possibility of accident occurrence. The data acquisition protocol was checked and permitted by the "Data Acquiring Ethics Evaluation Committee (DAEEC)" of Khulna University of Engineering \& Technology (KUET).

All information related to the experiment were verbally informed to the volunteers as well as regarding questions of the volunteer were also discussed elaborately so that they can understand the environment. Each volunteer sat on a comfortable armed chair during EEG data acquisition while they were requested to be focused on their assigned tasks. Afterward, for being accustomed to the experimental procedure, single trial data is taken before data recording for experimental purpose. To have a wide screen for a convenient 
environment for the volunteers, a projected screen was placed 8 feet away from the participants. The main tasks were of two kinds: $i$ ) Finding and counting specific alphabets from a paragraph and ii) Participating in virtual driving.

- Finding and Counting Specific Alphabet: This experiment was organized to compel each volunteer to pay consistent attention during the test. All volunteers were requested to find an exact alphabet from a given paragraph as soon as possible so that they might remain focused during the test time. Five alphabets (A, E, R, S, T) are requested to count as each counted as one trial.

- Participating in Virtual Driving: To simulate the driving environment and remain alert during test time a virtual racing (bike) game was supervised to play to the volunteers. In the game, 12 other competitors were available and consequently, the volunteer had to compete with others to continue his driving challenge as the real world. Difficulty level was selected as the expertise of the volunteers so that real driving environment would be copied and the volunteers were by default forced to remain concentrated during test time. They are requested not to fall and stay on their track.

In this research work, 14 male (age $=23 \pm 1.5$ ) right-handed volunteers were participated in previously explained two different protocols. Data acquisitions obeying the proposed protocols were performed in Neuroimaging Laboratory of Biomedical Engineering Department of Khulna University of Engineering \& Technology (KUET) shown in Fig. 1. The study protocols were previously permitted by the authority of the department. In the case of all data acquisition, no violation of the Helsinki Declaration was taken place.

\section{B. Data Acquisition}

During the protocol performed by the participants, EEG signals were acquired by B-Alert X10 devices (B-Alert Wireless EEG System, BIOPAC Systems Inc.). This device consists of the recording-transmit unit and receiving-data logging unit those are actually bi-directional transmission of digitized EEG signals. For data logging and transforming into further convenient format Acqknowledge (v4.4.1) software was used. A sensor headset cap contains 9 channel with EEG sensors located in the frontal (Fz, F3, and F4), central (Cz, C3, and $\mathrm{C} 4$ ) and parietal (Poz, P3, and P4) regions of the brain following the international 10-20 system. Electrode placement of this device and channel profiles are given in Fig. 2.

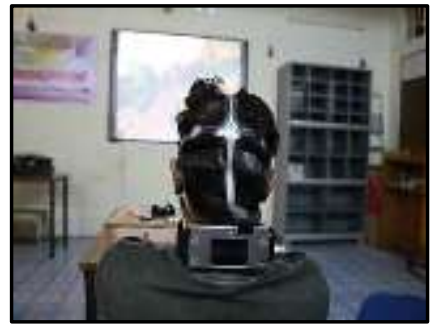

(a)

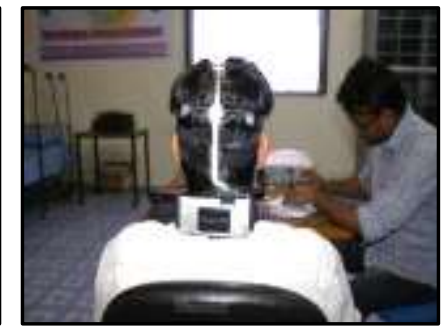

(b)
Fig. 1. Participants are Concentrating in Virtual Driving (a) and Alphabet Counting (b) during the EEG Data Acquisition Period.

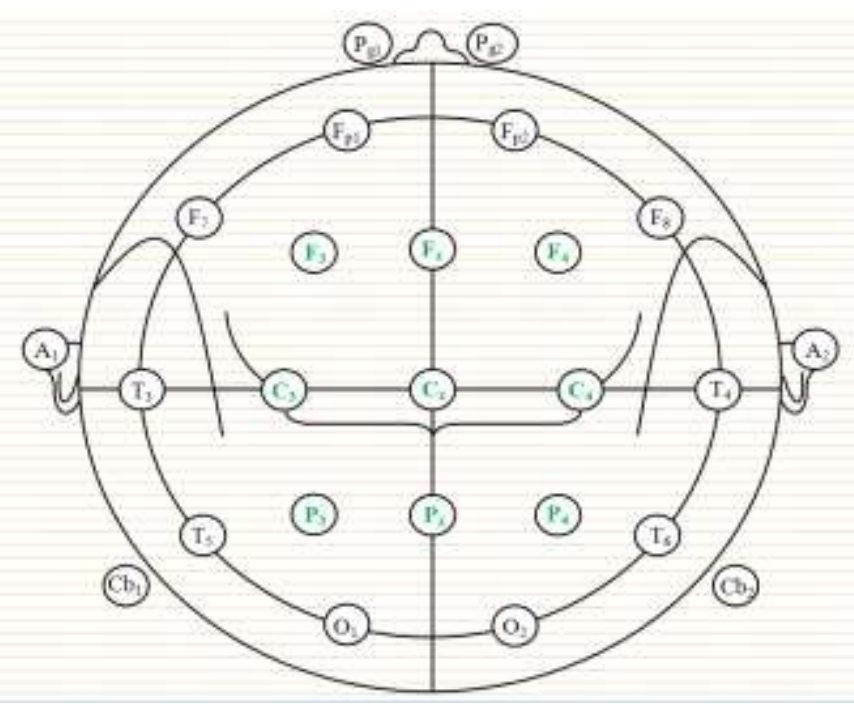

Fig. 2. The Locations of Concerned 9 Channel of Beta-Alert X-10 System (Green Colored) According to International 10-20 System.

This device is highly suitable for long time monitoring the cognitive state, such as engagement, workload, stress, confusion, drowsiness. Data acquisition was performed with the sampling rate of $256 \mathrm{~Hz}$ and before data acquisition; the impedances of the electrodes were checked to confirm satisfied conductivity.

\section{EEG Signal Filtering}

All raw EEG signal is filtered by a $50 \mathrm{~Hz}$ notch filter to remove the power line noise from the signal. After that bandpass Butterworth IIR filters are used to separate the alpha (8-13) $\mathrm{Hz}$ beta band (8-13) Hz, and theta band (4-7). The order of filter was selected optimum. We prepared an algorithm based on the method described in [30] to find the optimum filter order for the bandpass filter considering passband range, stopband range, sampling frequency, passband ripple, and stopband ripple. According to our algorithm, we used filter order 4 for the alpha band and 3 for beta and theta band [31]. In addition, the artifacts regarding eye blinking have been removed by the help of Acqknowledge (v4.4.1) software.

\section{Dimensionality Reduction using PCA}

Principal component analysis or PCA is a statistical transformation procedure to identify the patterns existed in data and expressing the data highlighting their similarities and differences. PCA is one of the most popular multivariate analytical methods and is necessary for all branches of scientific analysis. PCA can be applied in different purposes such as, to extract important information from big data set, for data compression, for simplification of the data description, to analyze the variable structures, etc. [32, 33]. In this paper, PCA is used for dimension reduction of EEG signal. There is a merit to use PCA to reduce the signal dimension of the EEG signal. Since there is a slight variation among the channels of the similar area due to its poor spatial resolution, PCA can find the maximum variation from the input higher dimensions. In addition, it also reduces the size of the feature vectors of the ANN input that leads it to gain higher classification accuracy. Therefore, for classifying purpose, three or more channels can 
be transformed into a single signal by PCA. In this work, the frontal area of the brain is covered by three channels, F3, F4, and Fz. If a matrix $\Lambda$ consists of the data of F3, F4, and Fz which means the data matrix, $\Lambda$ is of three dimensional. Now, a matrix $U$ can be calculated which represents the eigenvectors sorted as the eigenvalues of the covariance matrix of $\Lambda$. In that case, we can get the PCA transformation of the data $\Lambda$ in the form of $\mathrm{Y}$ as,

$Y=U^{T} \Lambda$

The eigenvectors are also termed as the principal components. If only first $r$ rows of $Y$ are selected to project the data, the data becomes of $r$ dimensional from $d$ dimensions. This transformation is performed by singular value decomposition (SVD). The procedure to perform PCA by SVD can be described by matrix decomposition. Suppose, the matrix, $\Lambda$ can be decomposed using SVD as [34],

$$
\Lambda=\Omega \Gamma \Psi^{T}
$$

Here, $\Omega$ is an $\mathrm{n} \times \mathrm{m}$ matrix with orthonormal columns ( $\left.\Omega^{T} \Omega=I\right), \Psi$ is an $m \times m$ orthonormal matrix $\left(\Psi^{T} \Psi=I\right)$, and $\Gamma$ is an m $\times \mathrm{m}$ diagonal matrix with positive or zero elements which is also known as singular value. Besides, we can calculate the covariance matrix, $\mathrm{C}$ of $\Lambda$ as,

$C=\frac{1}{N} \Lambda \Lambda^{T}=\frac{1}{N} \Omega \Gamma^{2} \Omega^{T}$

As the singular values are sorted in descending order and if $\mathrm{n}$ is less than $\mathrm{m}$, the first $\mathrm{n}$ columns in corresponds to the sorted eigenvalues of matrix $\mathrm{C}$. On the other hand, if $\mathrm{m}$ is greater than or equal $\mathrm{n}$, the first $\mathrm{m}$ corresponds to the sorted non-zero eigenvalues of $\mathrm{C}$. Eventually the transformed data can be presented as,

$Y=U^{T} \Lambda=U^{T} U \Gamma \Psi^{T}$

\section{E. Feature Extraction using DWT}

In case of the non-stationary signal, only frequency information is not enough as linear time-invariant system. According to the consideration of the non-stationary signal, it is necessary to observe the time vs frequency information. DWT is a technique for spectral analysis for the non-stationary signal which provides time-frequency information of the signal [35]. EEG signals are often considered as non-stationary signal and that's why DWT have been used by a number of notable research works [36-37]. DWT leads to getting fair timefrequency localization by providing wider windows at low frequencies and narrow windows at high frequencies. DWT can decompose a signal into suitable sub-bands by applying successive high-pass and low-pass filtering. The original time domain signal is first delivered through a half-band high-pass filter and a low-pass filter.

Wavelets are defined by two different functions those are termed as the wavelet function $\psi(\mathrm{t})$ (or mother wavelet) and scaling function $\varphi(\mathrm{t})$ (or father wavelet) in the continuous time domain. In case of discrete time domain, scaling function $\varphi_{j, k}[n]$ is used for low-pass filter and wavelet function, $\psi_{j, k}[n]$ are used for high pass filter as the relation given below, $\varphi_{j, k}[n]=2^{j / 2} h\left(2^{j} n-k\right)$

and

$\psi_{j, k}[n]=2^{j / 2} g\left(2^{j} n-k\right)$

Where, $\mathrm{n}=0,1,2, \ldots, M-1, \mathrm{j}=0,1,2, \ldots, \mathrm{J}-1, \mathrm{k}=0,1$, $2, \ldots, 2 j-1, J$ equals to $\log _{2}(M)$ and $M$ is the length of the signal and chosen as $2^{\mathrm{J}}$. Here, the function $h(\bullet)$ and $g(\bullet)$ are the corresponding impulse responses for low-pass and highpass filter, respectively. On the other hand, approximation coefficients $a_{i}(k)$ and detail coefficients $d_{i}(k)$ in $i^{\text {th }}$ level are equated as (3) \& (4), respectively.

$$
\begin{aligned}
& a_{i}(k)=\frac{1}{\sqrt{M}} \sum x[n] \varphi_{j, k}[n] \\
& d_{i}(k)=\frac{1}{\sqrt{M}} \sum x[n] \psi_{j, k}[n]
\end{aligned}
$$

for $k=0,1,2, \ldots . ., 2 j-1$. Scaling function and wavelet function of Daubechies-4 (db4) wavelet is used for the signal processing of this research work. Using Daubechies-4 (db4) wavelet, ten important features (maximum, minimum, mean, standard deviation, median, mean absolute deviation, median absolute deviation, max norm, 11 norm, and 12 norm) are extracted for classification purpose because these features contribute to attain higher classification accuracy $[4,7]$. Since the mathematical methods of calculating these features are very common, we have avoided to describe it, elaborately.

\section{F. Power Spectrum Density Estimation using Welch Method}

For random signals, it is only possible to propose probabilistic reports about the dissimilarity of the signals based on the probability of occurrence. To assess EEG signal PSD as a frequency domain feature provides crucial information about the distribution of power.

Power spectrum or spectral analysis of the signal $x(t)$ is the distribution of power over its frequency components. In this research work, beta PSD is calculated from each to point out the variation of PSD $\left(\mu \mathrm{V}^{2} / \mathrm{Hz}\right)$ according to the different tasks using the FFT algorithm. A random signal usually contains finite average power which is characterized as average power spectral density. The average power, $\mathrm{P}$ of the signal $x(t)$ during the total length of the signal period is defined as,

$$
P=\lim _{T \rightarrow \infty} \int_{-T}^{T}|x(t)|^{2} d t
$$

The mathematical relation given in (9) is for a continuous time signal. For discrete time signal, the notation $x(t)$ becomes $x(n)$ where $t=n T$ ( $T$ is sampling time interval and $\mathrm{n}$ is the sequence number). Therefore, for analyzing the frequency content of the discrete time signal, PSD is the Fourier transform of the auto-correlation function which can be represented as [5],

$$
P_{x}\left(e^{j \omega}\right)=\sum_{k=-\infty}^{\infty} r_{x}(k) e^{-j w w}
$$


In (10), $r_{x}(k)$ means autocorrelation for the periodic signal. But for the Ergodic process,

$$
r_{x}(k)=\operatorname{Lim}_{N \rightarrow \infty}\left\{\frac{1}{2 N+1} \sum_{n=-N}^{N} x(n+k) \otimes x(n)\right\}
$$

Where ' $\otimes$ ' denotes convolution of two signals $[5,38]$.

PSD calculation adopting windowing method is very important for nonparametric such as EEG signal. For nonparametric power spectral density estimation, the Welch method is most renowned method than the other methods (Periodogram and Bartlett). Let's suppose that the successive sequences are offset by $D$ points and that each sequence is $L$ point long, then the $i^{\text {th }}$ sequence is,

$x_{i}(n)=x(n+i D)$

Thus L-D points are overlapped. If entire $U$ data points are covered by $K$ sequences then,

$N=L+D(K-1)$

According to the previous conditions, Welch's method is written as,

$$
\hat{P}_{w}\left(e^{j \omega}\right)=\frac{1}{K L U} \sum_{i=0}^{K-1}\left|\sum_{n=0}^{L-1} w(n) x(n+i D) e^{-j r w}\right|^{2}
$$
is,

Therefore, the expected value by Welch's estimation [37]

$$
E\left\{\hat{P}_{W}\left(e^{j \omega}\right)\right\}=E\left\{\hat{P}_{M}\left(e^{j \omega}\right)\right\}=\frac{1}{2 \pi L U} P_{x}\left(e^{j \omega}\right) \star\left|W\left(e^{j \omega}\right)\right|^{2}
$$

\section{G. Relative Power Index Estimation}

The absolute power (AP) of a frequency band is calculated by the summation of all the power values in its frequency range. Relative power (RP) for each band was originated through articulating AP in apiece frequency band as the percent of the AP over the two frequency bands. If any band relates to specific neural activities, its relative power also increases with respect to resting condition. Therefore, relative power plays important roles in finding the specific electrical activities from the EEG signal. In this research work, AP is calculated from 1 to $100 \mathrm{~Hz}(50 \mathrm{~Hz}$ already filtered by notch filter). Due to the aforementioned consequences, the RP is calculated as [5, 39],

$$
R P\left(\varphi_{1}, \varphi_{2}\right)=\frac{P\left(\varphi_{1}, \varphi_{2}\right)}{P(1,100)} \times 10 \varphi_{5}
$$

Here, $\mathrm{P}$ indicates the power, RP represents the Relative Power, and $\varphi 1 \& \varphi 2$ is the low and high frequency, respectively.

\section{H. Alertness Classification Methodology using ANN}

ANN is a prominent classifier for EEG signal classification in supervised learning technique. In ANN, know features of different data class are fed and trained it to make a predictive model to classify the unknown data feature [40]. According to the feature size and class number, the structure of ANN is set to train the network with some suitable hidden layers. In our work, $\mathrm{NN}$ is designed considering 10 inputs as 10 extracted features and 3 outputs are contemplated as 3 classes' $i$. $e$. resting with eyes open, resting with eyes closed and active state (Active state may be either virtual driving or alphabet counting mental state). In the hidden layer, different neuron numbers are employed but better results have been found within 10-14 neurons in our work. So in the hidden layer, 10 neurons are used. A model of the designed ANN with inputs, outputs, and hidden layer is given in Fig. 3.

In preprocessing step for feature extraction from the signal, three frontal channels (F3, Fz, and F4) are taken into consideration for the next step. These three signals of every class are analyzed with PCA and linearly transformed these signals into three principal components.

Only first principle component had been reserved for feature extraction. Afterward, each signal was transformed to DWT for statistical feature extraction and arranged as a feature vector. These feature vectors were feed to the ANN to evaluate the accuracy of the alertness detection for the individual participant. These steps from signal processing to classification can be represented briefly by flow diagram given in Fig. 4 . Feature vectors and targets are feed to the ANN with feedforward network using pattern recognition algorithm of MATLAB NN toolbox. All trials are then randomly distributed for training (70\%), testing (15\%), and validation (15\%).

\section{Statistical Method to Detect Alertness}

ANN needs a set of features to take a decision and this process is quite complex. Additionally, machine learning related hardware design is costly. Due to this bargain, we have proposed a different method based on the statistical interpretation which can be more efficient to detect alertness state with compared to resting states. To plan such a methodology, first of all, a wide statistical survey has been performed to find the most significant features those can be distinguished different mental states. From that survey, it can be concluded that spectral density varies significantly with the variation of mental states. For the hypothetical test on the previous statement, we calculated RP of alpha, beta, delta, and theta band of EEG signal and based on the ANOVA we found that only beta RP can significantly distinguish the mentioned mental states from EEG signal. Therefore, for alertness monitoring, beta RP is taken into account to identify the average level of PSD values for each mental task. From the different values of PSD for each individual mental state, it is very easy to consider a threshold value to alert for nonalertness. The stepwise procedures of such signal processing are briefly presented by the block diagram given by Fig. 5 . It is also mentionable that in case of driving, this algorithm can be used to alert drivers while their mental consciousness will reach below the threshold value. 


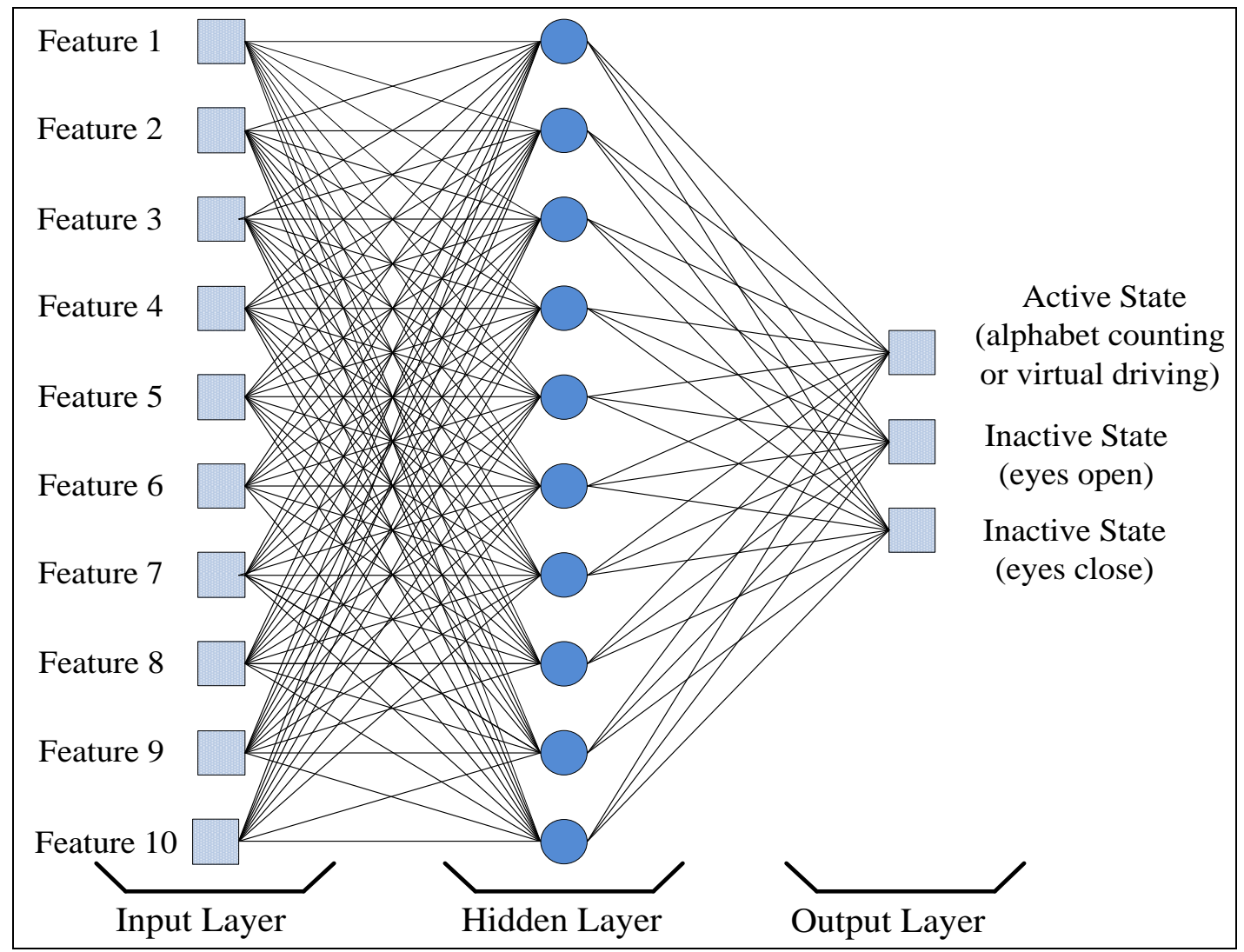

Fig. 3. The ANN Model for Alertness Classification with 10 Neurons in Hidden Layers and Three Output Layers.

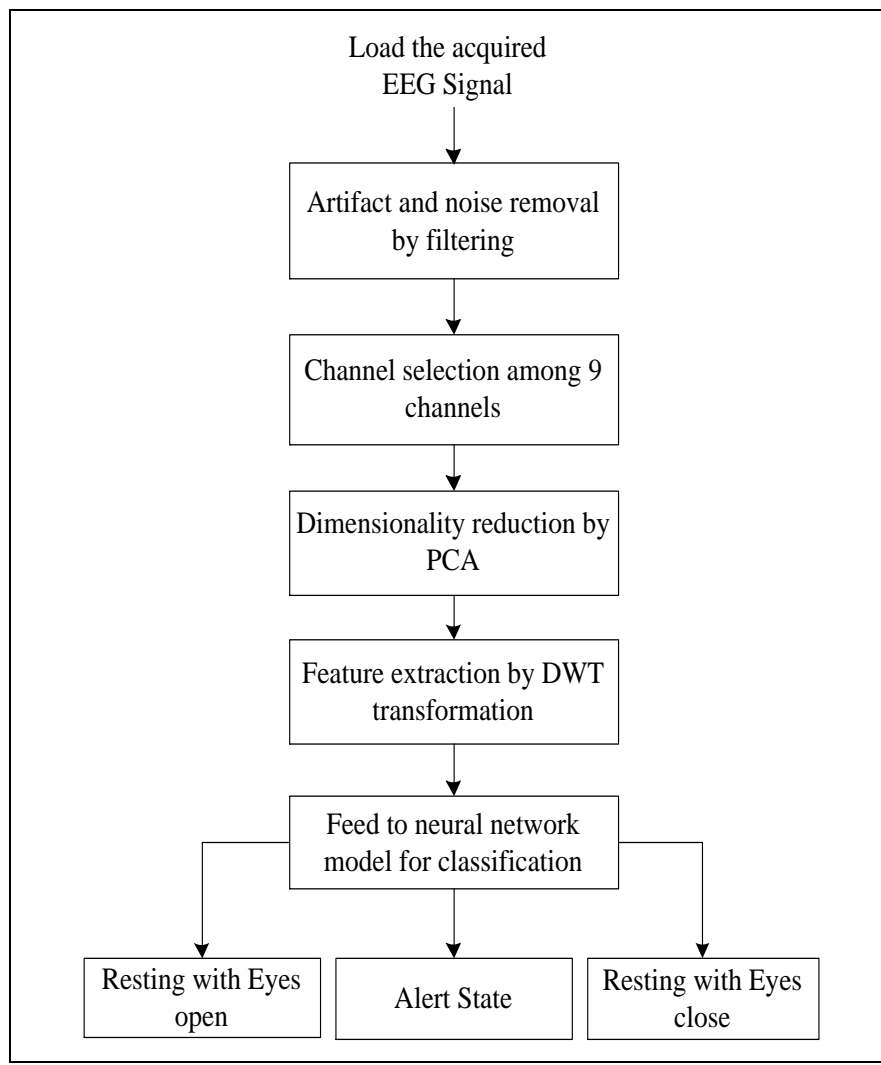

Fig. 4. Flow Diagram of the Mental Alertness Classification using ANN.

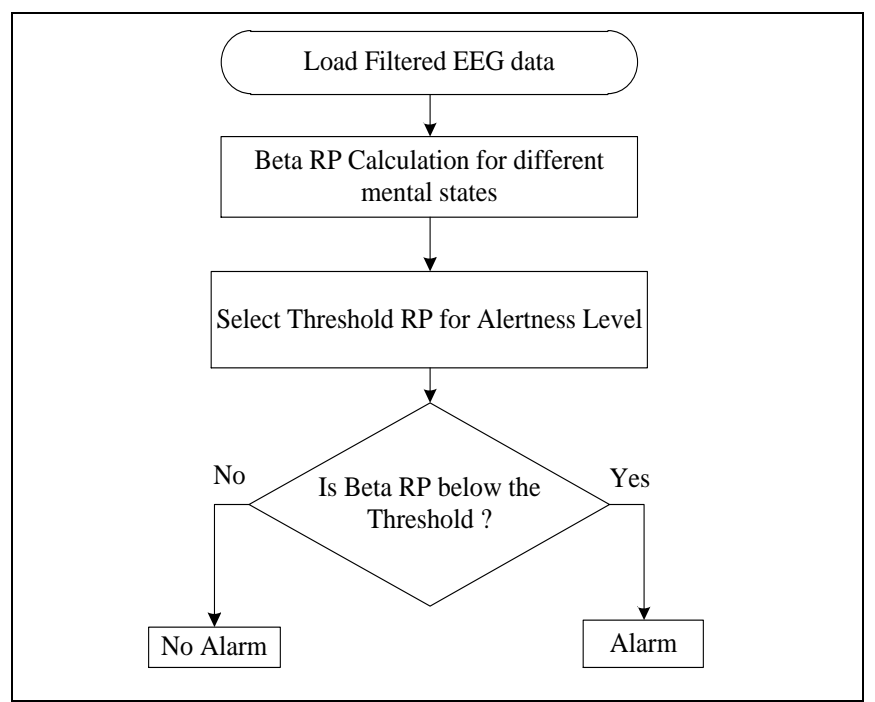

Fig. 5. Flow Diagram of the Mental Alertness Monitoring based on a Statistical Threshold.

\section{EXPERIMENTAL RESUlTS AND DisCUSSIONS}

In this research work, data acquisitions were performed based on four different mental states. Among them, two mental states are protocoled to highlight the alertness of mental state or concentrated in any task. One of them was to count a specific alphabet from a paragraph with full concentration and in another task, volunteers participated in virtual driving. The other two tasks are considered as mental control states and 
those are resting conditions with eyes open and eyes closed. The concentration level in some defined works like alphabet counting and virtual driving should have higher than the resting states (either at eyes open or eyes closed). Our goal of this work was to classify the alertness while being engaged of doing the different task with respect to resting state and monitor this alertness state of the brain from EEG signal and the level of alertness for different mental states of the brain.

\section{A. Alertness Classification Results}

Classification is accomplished according to three class classification by differing alphabet counting and virtual driving with resting state. For that purpose statistical features collected from DWT are organized according to $3 \times 3$ feature vector and feed to the ANN. The classification results are shown in Table 1 . of the 14 volunteers. Average accuracy is calculated by taking 5 consecutive accuracies and highest accuracy is the highest one among those 5 accuracies. From the table, it's noticeable that $100 \%$ accuracy frequently reappears though lowest accuracies are also enlarged. After analyzing all those results we can conclude that without taking all channels, only taking responsible channels with corresponding lobes that represents rhythmic changes with a specific task. Additionally, in this result, PCA also played an important role depending on the single signal by combining principal components of all signals. Closely or importantly related to our works are shortly presented by Table 2 with their protocol and classification efficiency. The results of the previous works presented in this table help to prove the effectiveness of the proposed protocol and classification methods of this work. For classifying among alphabet counting and resting state (eyes close and open), the lowest average accuracy is $72 \%$ of five consecutive results and from the highest accuracy, $86.70 \%$ is the lowest. For virtual driving and resting state (eyes close and open), the lowest average accuracy is $76 \%$ of five consecutive results, from the highest accuracy, $93.3 \%$ is the lowest. By adopting this method alertness state can be classified effectively.

\section{B. Alertness Monitoring}

It is already mentioned that to monitor alertness from mental states of corresponding EEG signals a wide survey was performed. To achieve this goal, first of all, it is necessary to find out one of the major features of EEG signal that can be able to differentiate the mental states of different tasks. It is already mentioned that mental alertness significantly increases the power of the beta band. Therefore, all the relative powers of the beta band from all the participants for all the tasks are calculated from power spectral density (PSD) by Welch method. It can be noted that the relative power of beta or beta relative power is the ratio of PSD of the beta band $(13-30 \mathrm{~Hz})$ and PSD of total EEG signal (1-49Hz). Therefore, beta relative power is a unitless quantity. The beta relative power of 10 participants among 14 for four different tasks is calculated and tabulated as following below. In the case of the other four participants, we got some unusual results and hence these results are excluded. Probably these subjects are BCI ignorant. Table 3, Table 4, and Table 5 are presenting the beta RP of the eyes close, eyes open, and alert conditions (alphabet counting and virtual driving) of all channels, respectively.

TABLE I. THE RESULTS OF THE CLASSIFICATION ACCURACY OF THE INDIVIDUAL PARTICIPANTS

\begin{tabular}{|c|c|c|c|c|}
\hline \multirow{2}{*}{$\begin{array}{l}\text { Participants } \\
\text { ID }\end{array}$} & \multicolumn{2}{|c|}{$\begin{array}{l}\text { Alphabet counting and resting state } \\
\text { (eyes close and open) }\end{array}$} & \multicolumn{2}{|c|}{$\begin{array}{l}\text { Virtual driving and resting state } \\
\text { (eyes close and open) }\end{array}$} \\
\hline & Average Accuracy (\%) & Highest Accuracy (\%) & Average Accuracy (\%) & Highest Accuracy (\%) \\
\hline P1 & 78.66 & 95 & 90.64 & 96 \\
\hline $\mathrm{P} 2$ & 81.5 & 91 & 76 & 93.3 \\
\hline P3 & 94.66 & 89 & 82 & 91 \\
\hline P4 & 98.66 & 94 & 93.34 & 95.5 \\
\hline P5 & 80.02 & 86.70 & 93.34 & 95.5 \\
\hline P6 & 82.66 & 93.3 & 84 & 91.5 \\
\hline P7 & 98.66 & 91.5 & 93.32 & 96.66 \\
\hline P8 & 72 & 93.3 & 90.66 & 95 \\
\hline P9 & 88 & 98 & 89.34 & 91 \\
\hline $\mathrm{P} 10$ & 86.68 & 92 & 90.66 & 93.3 \\
\hline P11 & 82.56 & 94 & 82.68 & 93 \\
\hline $\mathrm{P} 12$ & 97.32 & 98 & 92 & 97 \\
\hline P13 & 94.66 & 88.5 & 98.66 & 100 \\
\hline P14 & 89 & 95 & 97.32 & 100 \\
\hline
\end{tabular}


TABLE II. THE COMPARATIVE RESUlts OF THE PROPOSED AND THE EXISTING WORKS

\begin{tabular}{|c|c|c|c|}
\hline Authors & Different Class & Methods and Features & Performance \\
\hline T. d. Silveira [9] & Awake or Drowsy States & DWT significant $\mathrm{m}$-term approximation & PhysioNet Sleep Database tested Accuracy $98.7 \%$ \\
\hline N. H. Liu [16] & $\begin{array}{l}\text { Attentiveness and } \\
\text { Inattentiveness }\end{array}$ & FFT and SVM and PSD of different bands & The accuracy of up to $76.82 \%$. \\
\hline Z. Mardi [17] & Sleepiness and Alertness & ANN Chaotic features and logarithm of the energy & $83.3 \%$ and this accuracy \\
\hline A. G. Correa [18] & $\begin{array}{l}\text { Alertness and Drowsiness } \\
\text { Stages }\end{array}$ & $\begin{array}{l}\text { WT and ANN, LDA } \\
\text { Features from time and spectral analysis }\end{array}$ & $\begin{array}{l}87.4 \% \text { and } 83.6 \% \text { of alertness and drowsiness } \\
\text { correct detections rates }\end{array}$ \\
\hline M. K. Kiymik [19] & Alert, Drowsy, and Sleep & $\begin{array}{l}\text { DWT and ANN Classifier. } \\
\text { PSD of different bands }\end{array}$ & $\begin{array}{l}\text { The accuracy of the ANN was } 96 \pm 3 \% \text { alert, } 95 \pm \\
4 \% \text { drowsy and } 94 \pm 5 \% \text { sleep. }\end{array}$ \\
\hline N. Boonnak [21] & Drowsy and Alert & WT and ANN, Energy-based features & $90.27 \%$ of accuracy \\
\hline A. Subasi [22] & Alert, Drowsy, and Sleep & $\begin{array}{l}\text { DWT and MLPNN } \\
\text { Spectral features }\end{array}$ & $\begin{array}{l}\text { Classification rate was } 93.3 \% \text { alert, } 96.6 \% \text { drowsy, } \\
\text { and } 90 \% \text { sleep. }\end{array}$ \\
\hline M. K. Wali [24] & Driver Distraction Level & $\begin{array}{l}\text { DWPT, FFT and PNN Classifier, } k \text {-Nearest } \\
\text { Neighbor Classifier, Fuzzy Subtractive Clustering. } \\
\text { Spectral Centroid, and PSD }\end{array}$ & $\begin{array}{l}\text { The best average accuracy subtractive fuzzy } \\
\text { inference system classifier is } 79.21 \%\end{array}$ \\
\hline A. Gupta [25] & Mental Task & $\begin{array}{l}\text { EMD, WT, and LDC, QDC, kNN, and SVM } \\
\text { classifier. }\end{array}$ & Highest $95 \%$ \\
\hline L. J. Trejo [28] & Alert or Fatigued & $\begin{array}{l}\text { Kernel Partial Least Squares classifier. } \\
\text { PSD }\end{array}$ & 89.53 to $98.89 \%($ mean $=98.30 \%)$ \\
\hline Proposed Method & $\begin{array}{l}\text { Alertness and resting state } \\
\text { (eyes open and close) }\end{array}$ & $\begin{array}{l}\text { DWT, PCA, and ANN } \\
\text { Statistical features }\end{array}$ & $\begin{array}{l}\text { Highest Alertness Classification accuracy is } 100 \% \text {; } \\
\text { Lowest Alertness Classification accuracy is } 72 \% \text {. }\end{array}$ \\
\hline
\end{tabular}

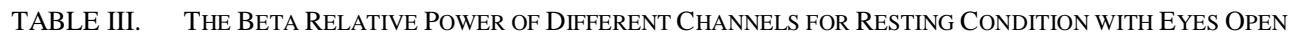

\begin{tabular}{|l|l|l|l|l|l|l|l|l|l|}
\hline \multirow{2}{*}{ Participants } & \multicolumn{9}{l|}{ Channel No and their corresponding beta relative power } \\
\cline { 2 - 11 } & 1 & 2 & 3 & 4 & 5 & 6 & 7 & 8 \\
\hline P1 & 1.0476 & 0.4902 & 0.7719 & 0.8232 & 0.7351 & 0.5716 & 0.5512 & 1.4456 \\
\hline P2 & 1.3989 & 1.4084 & 1.4257 & 6.2653 & 4.1449 & 4.8885 & 4.3706 & 2.1826 & 0.9409 \\
\hline P3 & 0.3427 & 0.3328 & 0.3825 & 0.4236 & 0.4456 & 0.2906 & 0.3103 & 0.4462 & 0.3471 \\
\hline P4 & 0.4213 & 0.4178 & 0.4441 & 0.4984 & 0.5908 & 0.3481 & 0.3851 & 0.5543 & 0.4511 \\
\hline P5 & 1.1342 & 0.9803 & 1.1601 & 3.6770 & 3.6053 & 3.3989 & 2.3889 & 1.5635 & 1.5636 \\
\hline P6 & 1.3882 & 1.1782 & 1.2423 & 3.8851 & 2.9551 & 2.5713 & 2.1353 & 2.1727 \\
\hline P7 & 0.3902 & 0.3827 & 0.4661 & 0.5764 & 0.3743 & 0.5062 & 0.4236 & 0.4881 \\
\hline P8 & 0.4558 & 0.4512 & 0.4845 & 0.6306 & 0.4112 & 0.5636 & 0.4660 & 0.5541 & 0.3851 \\
\hline P9 & 0.1275 & 0.1314 & 0.1474 & 0.1498 & 0.2049 & 0.1554 & 0.2258 & 0.1134 \\
\hline P10 & 0.1389 & 0.1463 & 0.1752 & 0.1638 & 0.1508 & 0.2777 & 0.3138 & 0.1284 \\
\hline
\end{tabular}

TABle iV. The Beta Relative Power of DifFerent Channels For Resting Condition with Eyes Closed

\begin{tabular}{|l|l|l|l|l|l|l|l|l|l|}
\hline \multirow{2}{*}{ Participants } & \multicolumn{9}{l|}{ Channel No and their corresponding beta relative power } \\
\cline { 2 - 10 } & 1 & 2 & 3 & 4 & 5 & 6 & 7 & 8 \\
\hline P1 & 1.5127 & 1.1171 & 0.9536 & 0.8689 & 0.7513 & 0.9247 & 0.7061 & 1.8218 \\
\hline P2 & 0.5472 & 0.5786 & 0.6109 & 1.3484 & 0.9312 & 0.8210 & 0.8609 & 0.7519 \\
\hline P3 & 0.3538 & 0.3631 & 0.3180 & 0.3490 & 0.3642 & 0.2276 & 0.2424 & 0.4104 & 0.6947 \\
\hline P4 & 0.3763 & 0.3843 & 0.3618 & 0.3898 & 0.4069 & 0.2650 & 0.2858 & 0.4424 \\
\hline P5 & 0.7105 & 0.7426 & 0.9331 & 2.8111 & 1.4212 & 1.5013 & 1.1009 & 1.0637 \\
\hline P6 & 0.7173 & 0.7647 & 0.8211 & 2.2719 & 1.1071 & 1.8593 & 1.0097 & 1.0562 & 0.3665 \\
\hline P7 & 0.4142 & 0.4069 & 0.5653 & 0.6067 & 0.4071 & 0.5438 & 0.4991 & 0.5206 \\
\hline P8 & 0.4491 & 0.4419 & 0.5438 & 0.5886 & 0.4176 & 0.5267 & 0.4844 & 0.5516 & 0.3984 \\
\hline P9 & 0.2682 & 0.2869 & 0.3048 & 0.2609 & 0.2357 & 0.2404 & 0.2216 & 0.2343 \\
\hline P10 & 0.2841 & 0.3051 & 0.3449 & 0.2844 & 0.2674 & 0.2464 & 0.2473 & 0.2426 \\
\hline
\end{tabular}


TABLE V. The Beta Relative Power of DifFERENT CHANNEls For AlPhabet Counting

\begin{tabular}{|l|l|l|l|l|l|l|l|l|l|}
\hline \multirow{2}{*}{ Participants } & \multicolumn{6}{|l}{ Channel No and their corresponding beta relative power } \\
\cline { 2 - 10 } & 1 & 2 & 3 & 4 & 5 & 6 & 7 & 8 \\
\hline P1 & 1.3101 & 0.8491 & 0.8473 & 0.8724 & 0.7141 & 0.9586 & 0.8339 & 1.8886 \\
\hline P2 & 0.3319 & 0.2091 & 0.2667 & 0.2462 & 0.5977 & 0.3659 & 0.2838 & 0.4281 & 1.1220 \\
\hline P3 & 0.2572 & 0.2536 & 0.2765 & 0.3307 & 0.3711 & 0.2089 & 0.2299 & 0.3335 \\
\hline P4 & 0.3890 & 0.3839 & 0.3892 & 0.4488 & 0.5362 & 0.3070 & 0.3413 & 0.5056 & 0.2771 \\
\hline P5 & 1.2802 & 1.2989 & 0.6829 & 1.2513 & 0.8462 & 2.1024 & 4.3659 & 1.7514 & 0.4258 \\
\hline P6 & 1.2646 & 1.2471 & 0.7856 & 2.3043 & 1.2650 & 2.5678 & 3.9286 & 2.0101 \\
\hline P7 & 0.5088 & 0.5072 & 0.5145 & 0.6749 & 0.4449 & 0.5464 & 0.5862 & 0.6097 & 0.9911 \\
\hline P8 & 0.3631 & 0.3560 & 0.3760 & 0.4443 & 0.3189 & 0.4025 & 0.3778 & 0.4068 \\
\hline P9 & 0.1377 & 0.1461 & 0.1516 & 0.1355 & 0.1301 & 0.1599 & 0.2553 & 0.1209 & 0.3286 \\
\hline P10 & 0.1278 & 0.1352 & 0.1492 & 0.1994 & 0.1441 & 0.1759 & 0.2270 & 0.1702 \\
\hline
\end{tabular}

Based on the results of beta RP of different functional brain states of different positional EEG signals were statistically analyzed by one-way and two-way ANOVA considering $95 \%$ confidence interval. The results are given in Table 6 and Table 7. This result helped us to take a decision on the feature we can trust. From the one way ANOVA, we get that statistical difference among the mental states are strongly significant $(p<0.01)$. It is also found that the beta RP level will definitely vary with the variation of participants because the two way ANOVA is analyzed considering events versus participants and get the significance level as strongly convincing $(p 1<0.05 \&$ $p 2<0.001)$. Therefore one way and two way ANOVA results are too convincing to consider the beta RP to differentiate mental alertness than the other two control states. To check the feasibility of this feature to distinguish the mental alertness conditions from EEG signal, we acquired a set of EEG signals from the participants with multiple tasks in one-time interval like virtual driving, resting condition with eyes open and eyes closed, and alphabet counting, simultaneously. These combined task EEG signals are tested with our proposed algorithm as previously described. The results of a typical participant are given regarding all channels in Fig. 6. The results depict that the electrodes of frontal and central areas are giving notable variation according to the tasks.

TABLE VI. RESULtS OF ONE WAY ANOVA

\begin{tabular}{|c|c|c|c|c|}
\hline Source of Variance & Degrees of Freedom & Factor & p-value & F Critical \\
\hline Channel 1 & \multirow{9}{*}{3} & 5.206473586 & 0.004330514 & \multirow{9}{*}{2.866265551} \\
\hline Channel 2 & & 5.399106146 & 0.003582388 & \\
\hline Channel 3 & & 7.225764165 & 0.000645253 & \\
\hline Channel 4 & & 4.643599552 & 0.007614366 & \\
\hline Channel 5 & & 5.665255929 & 0.002764523 & \\
\hline Channel 6 & & 6.019326235 & 0.001968346 & \\
\hline Channel 7 & & 5.074147715 & 0.004938149 & \\
\hline Channel 8 & & 4.104782628 & 0.013258564 & \\
\hline Channel 9 & & 6.361304704 & 0.001425527 & \\
\hline
\end{tabular}

TABLE VII. RESULtS OF Two WAY ANOVA

\begin{tabular}{|c|c|c|c|c|}
\hline Source of Variance & Degrees of Freedom & Factor & p-value & F Critical \\
\hline Channel 1 & \multirow{9}{*}{3} & 5.206473586 & 0.004330514 & \multirow{9}{*}{2.866265551} \\
\hline Channel 2 & & 5.399106146 & 0.003582388 & \\
\hline Channel 3 & & 7.225764165 & 0.000645253 & \\
\hline Channel 4 & & 4.643599552 & 0.007614366 & \\
\hline Channel 5 & & 5.665255929 & 0.002764523 & \\
\hline Channel 6 & & 6.019326235 & 0.001968346 & \\
\hline Channel 7 & & 5.074147715 & 0.004938149 & \\
\hline Channel 8 & & 4.104782628 & 0.013258564 & \\
\hline Channel 9 & & 6.361304704 & 0.001425527 & \\
\hline
\end{tabular}



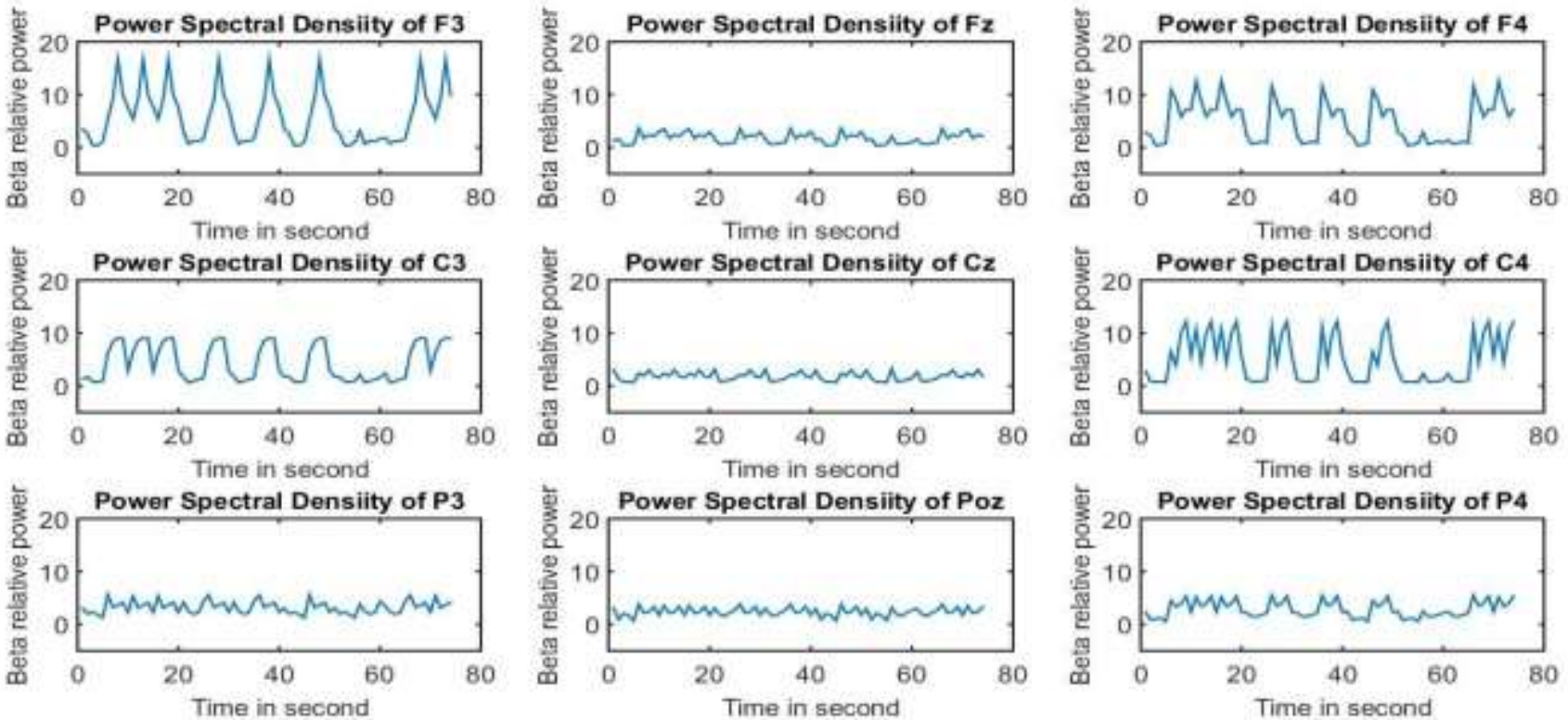

Fig. 6. Variation in beta RP of the EEG Signal Regarding the Combinational Tasks in all 9 Channels that Exhibits the Alertness Condition of the Brain.
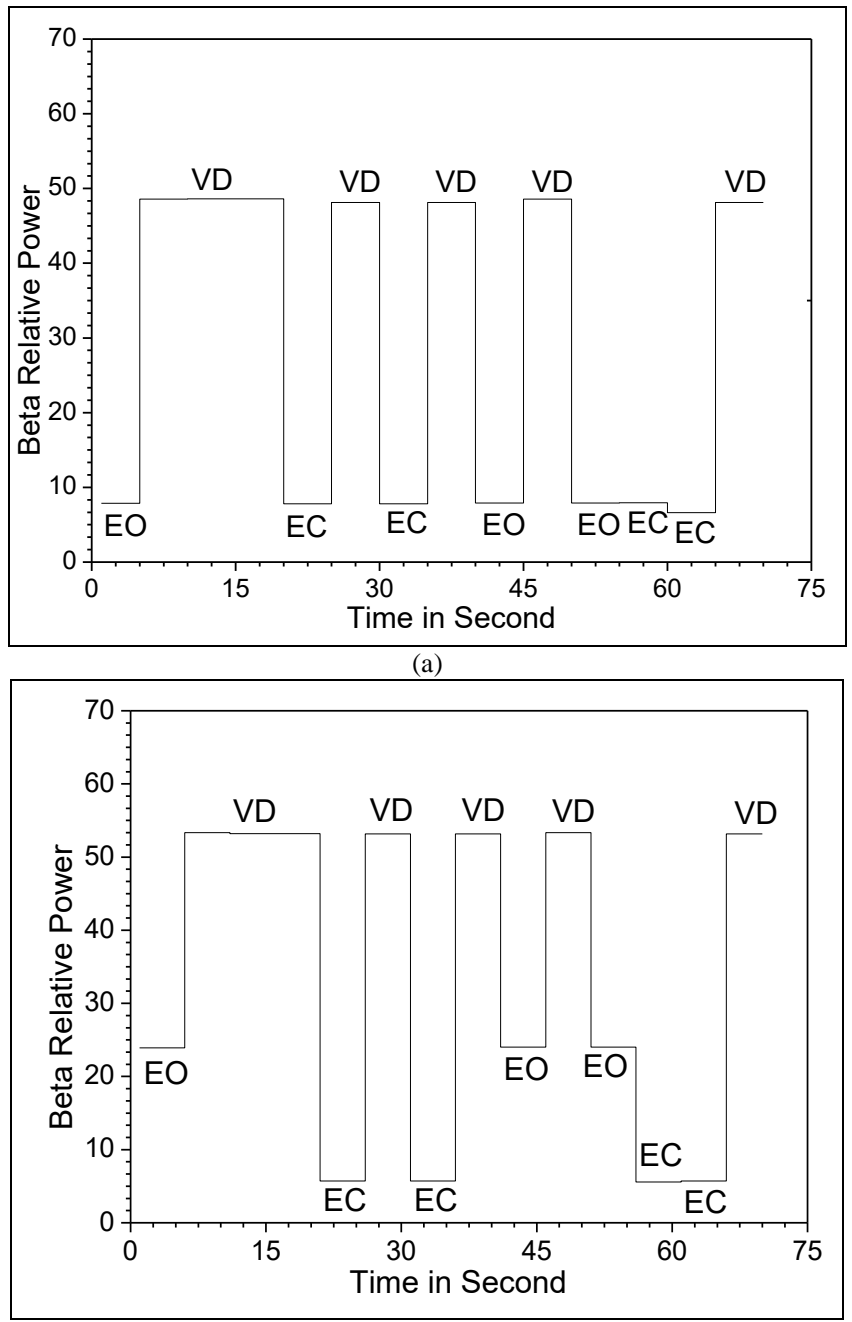

(b)

Fig. 7. Beta RP Variation with Time and the Task of Participants 1 (a) and Participants 2 (b).
Since our intention was to monitor alertness state, we focused on the frontal electrodes (F3, Fz, and F4). Among the three channels $(\mathrm{F} 3, \mathrm{Fz}, \mathrm{F} 4)$ for alertness monitoring, we found more impact on the F3 channel, consistently. So we propose with supporting results that $\mathrm{F} 3$ channel is promising and shows a responsible variation of the beta RP for alertness detection. The average beta RP's of two participants regarding the combined task is given in Fig. 7. The signals used in this algorithm is a combination of virtual driving, eyes open, and eyes close as mentioned in Table 8. The distributions of the beta RP of the two participants are plotted in Fig. 7 regarding the data of their F3 channels. From the figure, it is decipherable the transition of an alert state in comparison with resting state and the value of beta RP varies with subject to subject.

TABLE VIII. PROPOSED THRESHOLD VALUES FOR THE DIFFERENT PARTICIPANTS FOR ALERTNESS MONITORING DURING VIRTUAL DRIVING

\begin{tabular}{|l|l|l|l|l|}
\hline Subjects & $\begin{array}{l}\text { Average } \\
\text { RP of } \\
\text { EO }\end{array}$ & $\begin{array}{l}\text { Average } \\
\text { RP of EC }\end{array}$ & $\begin{array}{l}\text { Average } \\
\text { RP of } \\
\text { (VD) }\end{array}$ & $\begin{array}{l}\text { Proposed Threshold } \\
\text { Range } \\
\text { (Average RP) }\end{array}$ \\
\hline P1 & 5 & 7 & 52 & $20-40$ \\
\hline P2 & 20 & 5 & 36 & $25-30$ \\
\hline P3 & 5 & 1.5 & 10 & $7-8$ \\
\hline P4 & 1.9 & 1.7 & 2.4 & $2.1-2.2$ \\
\hline P5 & 7 & 10 & 36 & $15-30$ \\
\hline P6 & 21 & 11 & 35 & $26-30$ \\
\hline P7 & 2.5 & 2.5 & 18 & $10-15$ \\
\hline P8 & 4 & 4 & 58 & $20-40$ \\
\hline P9 & 1 & 1.25 & 5.5 & $3-4$ \\
\hline P10 & 1 & 1.1 & 10 & $5-8$ \\
\hline
\end{tabular}


By selecting a threshold value between the intervals of the beta RP value of alertness state and resting state, it is possible to monitor the human concentration level. More difference in beta RP value will provide benefits such as it will reduce the chances of making an error in taking a decision. Table 8 shows the average RP of EO, EC, and VD and proposes a threshold range for alertness monitoring of the individual participants in this work. From Table 8, it is noticeable that P3, P4, and P9 have a very limited range of threshold value with $1,0.1$ and 1 , respectively. In addition, P1 and P8 participants have a wide range of threshold value of 20. This will support P1 and P8 for alertness monitoring without almost any error as there is less chance of merging with $\mathrm{EO}$ and $\mathrm{EC}$ values.

\section{CONCLUSION}

EEG signals reflect the status of our mental state. Human alertness monitoring is essential for performing a governed task efficiently. This research work provides an effective methodology for alertness monitoring and classification. The classification results are very promising for alertness classification using the EEG signal. For monitoring alertness, as threshold varies with respect to every user, to avoid or less error it is better to train user and after getting acceptance level of accuracy after several simulations then select the threshold value. Alertness monitoring for drivers or pilots may have unprecedented change by reducing the chances of road crashes that will save innumerable lives. Thus this proposed research work can be adopted in designing vehicles or in other sectors where alertness monitoring is out most important to reduce the degree of risk.

This work was solely designed as offline approach. According to the results we found in this work, this method can be applicable to design an online module to observe the mental alertness state of a driver so that the system can alert the driver in case of drowsiness. In addition, the proposed work can be applicable to classify the alertness level of the students in a class.

\section{ACKNOWLEDGMENT}

This work was supported by the Higher Education Quality Enhancement Project (HEQEP), UGC, Bangladesh; under Subproject "Postgraduate Research in BME", CP\#3472, KUET, Bangladesh.

\section{REFERENCES}

[1] R. Xiao, J. S. Tokeshi, D. L. Vanderbilt, and B. A. Smith, "Electroencephalography power and coherence changes with age and motor skill development across the first half year of life," PLoS One, vol. 13, no. 1, pp. 1-17, 2018.

[2] Yuyi, Zhaoyun, L. Surui, S. Lijuan, L. Zhenxin, and D. Bingchao, "Motor imagery EEG discrimination using Hilbert-Huang entropy," Biomedical Research, vol. 28, no. 2, pp. 727-733, 2017.

[3] M. Fira, L. Goras, and Anca Lazar, "On P300 detection using scalar products," International Journal of Advanced Computer Science and Applications, vol. 9, no. 1, 2018.

[4] M. M. Rashid and M. Ahmad, "Multiclass motor imagery classification for BCI application", Proceedings of the International Workshop on Computational Intelligence (IWCI 2016), Jahangirnagar University (JU), 12-13 December 2016.

[5] F. Khanam, M. A. Rahman, and M. Ahmad, "Evaluating alpha relative power of EEG signal during psychophysiological activities in salat,"
International Conference on Innovations in Science, Engineering and Technology (ICISET), 27-28, 2018, Bangladesh, pp. 1-06.

[6] C. J. Lin, C. Wu, and W. A. Chaovalitwongse, "Integrating human behavior modeling and data mining techniques to predict human errors in numerical typing," IEEE Transactions on Human-Machine Systems, vol. 45, no. 1, February 2015.

[7] M. M. Rashid and M. Ahmad, "Epileptic seizure classification using statistical features of EEG signal", Proceedings of the International Conference on Electrical, Computer and Communication Engineering (ECCE 2017), CUET, 16-18 February 2017.

[8] R. P. Balandong, R. F. Ahmad, M. N. Mohamad Saad and A. S. Malik, "A Review on EEG-Based Automatic Sleepiness Detection Systems for Driver," IEEE Access, vol. 6, pp. 22908-22919, 2018.

[9] T. d. Silveira, A. d. J. Kozakevicius and C. R. Rodrigues, "Drowsiness detection for single channel EEG by DWT best m-term approximation," Research on Biomedical Engineering, vol. 31, no. 2, pp. 107-115, 2015.

[10] A. Hashemi, V. Saba, and S. N. Resalat, "Real time driver's drowsiness detection by processing the EEG signals stimulated with external flickering light," Basic and Clinical Neuroscience, vol. 5, no. 1, 22-27, 2014.

[11] http://asirt.org/initiatives/informing-road-users/road-safety-facts/roadcrash-statistics.

[12] E. G. Willcutt, "The prevalence of DSM-IV attentiondeficit/hyperactivity disorder: a meta-analytic review," Neurotherapeutics, vol. 9, no. 3, pp. 490-499, Neurother 2012.

[13] S. J. Kooij, S. Bejerot, A. Blackwell, H. Caci et al., "European consensus statement on diagnosis and treatment of adult ADHD: The European Network Adult ADHD," BMC Psychiatry. vol. 10, no. 67, 2010.

[14] S. Bálint, P. Czobor, A. U. Meszaros, V. Simon, and I. Bitter, "Neuropsychological impairments in adult attention deficit hyperactivity disorder: A literature review," Psychiatria Hungarica (in Hungarian), vol. 23, no. 5, pp. 324-335, 2008.

[15] Y. Ginsberg, J. Quintero, E. Anand, M. Casillas, and H. P. Upadhyaya, "Underdiagnosis of attention-deficit/hyperactivity disorder in adult patients: a review of the literature, "Journal of Clinical Psychiatry, vol. 16, no. 3, 2014.

[16] N. H. Liu, C. Y. Chiang, and H. C. Chu, "Recognizing the degree of human attention using EEG signals from mobile sensors," Sensors, vol. 13 no. 8, pp. 10273-10286, 2013.

[17] Z. Mardi, S. N. M. Ashtiani, and M. Mikaili, "EEG based drowsiness detection for safe driving using chaotic features and statistical tests," Journal of Medical Signals and Sensors, vol. 1, no. 2, pp. 130-137, 2011.

[18] A. G. Correa, L. Orosco, and E. Laciar, "Automatic detection of drowsiness in EEG records based on multimodal analysis," Medical Engineering \& Physics, vol. 36, no. 2, pp. 244-249, 2014.

[19] M. K. Kiymik, M. Akin, and A. Subasi, "Automatic recognition of alertness level by using wavelet transform and artificial neural network," Journal of Neuroscience Methods, vol. 139, no. 2, pp. 231-240, 2004.

[20] R. Kianzad and H. M. Kordy, "Automatic sleep stages detection based on EEG signals using combination of classifiers," Journal of Electrical and Computer Engineering Innovations, vol. 1, no. 2, pp. 99-105, 2013.

[21] N. Boonnak, S. Kamonsantiroj, and L. Pipanmaekaporn, "Wavelet transform enhancement for drowsiness classification in EEG records using energy coefficient distribution and neural network," International Journal of Machine Learning and Computing, vol. 5, no. 4, 2015.

[22] A. Subasi, M. K. Kiymik, M. Akin, and O. Erogul, "Automatic recognition of vigilance state by using a wavelet-based artificial neural network," Neural Computing and Applications, vol. 14, no. 1, pp. 45 $55,2005$.

[23] G. L. and W. Y. Chung, "Detection of driver drowsiness using wavelet analysis of heart rate variability and a support vector machine classifier," Sensors, vol. 13, no. 12, pp. 16494-511, 2013.

[24] M. K. Wali, M. Murugappan, and B. Ahmmad, "Wavelet packet transform based driver distraction level classification using EEG," Mathematical Problems in Engineering, 2013. 
[25] A. Gupta and D. Kumar, "Fuzzy clustering-based feature extraction method for mental task classification," Brain Informatics, vol. 4, no. 2, pp. 135-145, 2016.

[26] A. Gupta, R. K. Agrawal, and B. Kaur, "Performance enhancement of mental task classification using EEG signal: a study of multivariate feature selection methods," Soft Computing, vol. 19, no. 10, pp 2799$2812,2015$.

[27] D. Begum, K. M. Ravikumar, J. Mathew, S. Kubakaddi, and R. Yadav, "EEG based patient monitoring system for mental alertness using adaptive neuro-fuzzy approach," Journal of Medical and Bioengineering, vol. 4, no. 1, 2015.

[28] L. J. Trejo, K. Kubitz, R. Rosipal, R. L. Kochavi, and L. D. Montgomery, "EEG-based estimation and classification of mental fatigue," Psychology, vol. 6, no. 5, pp. 572-589, 2015.

[29] N. Gurudath and H. B. Riley, "Drowsy driving detection by EEG analysis using wavelet transform and k-means clustering," Procedia Computer Science, vol. 34, pp. 400-409, 2014.

[30] M. M. Hasan, M. H. A. Sohag, M. E. Ali, and M. Ahmad, "Estimation of the most effective rhythm for human identification using EEG signal," Proceedings of 9th International Conference on Electrical and Computer Engineering (ICECE), December 2016.

[31] J. G. Proakis and D. G. Manolakis, "Digital Signal Processing: Principles, Algorithms, and Applications," Third edition, Prentice-Hall of India Private limited, New delhi, 2002.

[32] H. Abdi and L. J. Williams, "Principal component analysis, " John Wiley \& Sons, Inc., vol. 2, July/August 2010.

[33] M. A. Rahman, M. M. Haque, A. Anjum, M. N. Mollah, and M. Ahmad, "Classification of motor imagery events from prefrontal hemodynamics for BCI application," International Joint Conference on Computational Intelligence (IJCCI), 14-15 December 2018, Bangladesh. pp. 1-06.

[34] I. T. Jolliffe, "Principal Component Analysis," Second Edition, Springer, 2002.

[35] U. Orhan, M. Hekim, and M. Ozer, "EEG signals classification using the $k$-means clustering and a multilayer perceptron neural network model," Expert Systems with Applications, vol. 38, pp. 13475-13481, 2011.

[36] A. R. Aguinaga and M. A. Lopez Ramırez, and M. R. B. Flores, "Classification model of arousal and valence mental states by EEG signals analysis and Brodmann correlations," International Journal of Advanced Computer Science and Applications, Vol. 6, No. 6, 2015.

[37] H. Djaghloul, J. Jessel, M. Batouche, and A. Benhocine, "Wavelet/PSObased segmentation and marker-less tracking of the gallbladder in monocular calibration-free laparoscopic cholecystectomy," International Journal of Advanced Computer Science and Applications, vol. 9, no. 7, 2018.

[38] E. C. Ifeachor and B. W. Jervis, Digital Signal Processing: A Practical Approach, Addison Wesley Publishers Ltd.

[39] B. Zhijie, L. Qiuli, W. Lei, L. Chengbiao, Y. Shimin, and L. Xiaoli, "Relative power and coherence of EEG series are related to amnestic mild cognitive impairment in diabetes," Frontiers in Aging Neuroscience, vol. 6, no. 11, February 2014.

[40] R. Ahmmed, M. A. Rahman, and M. F. Hossain, "An advanced algorithm combining SVM and ANN classifiers to categorize tumors with position from brain MRI images," Advances in Science, Technology and Engineering Systems Journal, vol. 3, no. 2, pp. 40-48, March 2018. 